\title{
Hydro-Mechanical Neonatal Encephalopathy (HNE): An Alternative Hypothesis
}

\section{Talbert DG*}

Institute of Reproductive and Developmental Biology, Imperial College School of Medicine, Queen Charlotte's Hospital, UK

*Corresponding author: Talbert DG, Institute of Reproductive and Developmental Biology, Imperial College School of Medicine, Queen Charlotte's Hospital, UK, Tel: 0208969 8151; E-mail: d.talbert@imperial.ac.uk

Rec Date: January 22, 2018; Acc Date: February 06, 2018, 2018; Pub Date: February 10, 2018

Citation: Talbert DG (2018) Hydro-Mechanical Neonatal Encephalopathy (HNE): An Alternative Hypothesis. J Neurol Neurosci Vol.9 No.1:245.

\section{Abstract}

Background: The World Health Organisation estimates that 4 million neonatal deaths occur yearly due to perinatal asphyxia, representing $38 \%$ of deaths of children under 5 years of age. Typically, neonatal encephalopathy occurs unexpectedly following an otherwise uneventful pregnancy.

Studies have shown that neural damage after hypoxiaischemia is delayed for several hours and that treatment with prolonged moderate hypothermia reduces cerebral injury and improves neurological outcome. Moderate hypothermia for 72 hours, if started within 6 hours of birth, reduces the rate of death and disability seen at 18 months of age.

Current explanations concentrate on various maladies that might cause such profound injuries. This hypothesis proposes the existence of a mechanical form of trauma arising in the birthing process.

The hydro-mechanical hypothesis: The Hydro-Mechanical Hypothesis comes in two phases, an initial effusive phase, followed by an ischemic phase. The effusive phase occurs, and can only occur, during delivery. The full uterine contraction pressure appears across the walls of cerebral vessels as the head emerges, but the body and placenta are still subject to contraction pressure. This pressure rapidly drives fluid out of cerebral vasculature into the surrounding interstitium.

The ischemic phase follows delivery. The distending pressure is no longer present, but the interstitial pressure remains high, constricting vessels, particularly cerebral capillaries and venules. This temporary compression will only last until the excessive interstitial fluid has dispersed, but many neurons may die in the meantime. Hypothermia works by reducing the metabolic demand in the neurons so that they can survive, though not necessarily function, on a meagre gas exchange until the excess interstitial fluid has dispersed and normal blood flow is restored.

Conclusion: A hydro-mechanical form of Neonatal Encephalopathy is possible which has no connection with the preceding pregnancy. This form would be expected to benefit from appropriate hypothermic therapy.

Keywords: Neonate; Hypoxia; Ischemia; Labour; Contractions

\section{Introduction}

The terms Perinatal Asphyxia, Neonatal Asphyxia, Birth Asphyxia, Neonatal Encephalopathy, Hypoxic-ischemic Encephalopathy, all relate to the clinical observation [1] that fetuses that have apparently progressed normally may develop profound hypoxic injury during and following the birth process. This causes death or disability in 2-3 per 1000 cases and accounts for about $20 \%$ of all cases of cerebral palsy.

Many mechanisms [2-4] have been proposed, with corresponding variation of treatments, but the only protocol showing promise is the empirical one of cooling of the infant [5]. The mechanism initiating such damage cascades has not yet been determined. The possible involvement of intrauterine pressure during contractions does not appear to have previously been considered. That is the subject of this article.

\section{Methodology}

\section{The hydro-mechanical neonatal encephalopathy (HNE) hypothesis}

This hypothesis concerns the movement of Extra Cellular Fluid (referred to hereafter as Tissue Fluid) occurring during the birthing process. It is postulated that HNE comes in two phases, an initial mechanical trauma which happens during delivery, and a second Hypoxic-Ischemic injury phase that results from vascular derangement during delivery.

The initial mechanical phase requires access to uterine contraction pressure and so cannot occur before or after delivery. It can only happen during delivery. The second (ischemic) phase is temporary, awaiting the dispersal of profound edema set up in the first phase. The following indicates a typical predicted sequence. 


\section{Phase I: Mechanical Trauma}

\section{Initial pressurizing (Early labor, cervix still closed)}

Figure 1 is a re-plot of part of the example given in reference [6]. This is the intrauterine pressure during labor registered via a catheter inserted into the amniotic fluid. Between contractions the intrauterine pressure was slightly less than $20 \mathrm{mmHg}$, but during contractions it reached 100 $\mathrm{mmHg}$.

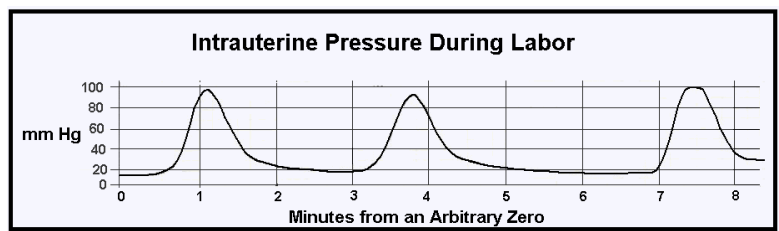

Figure 1 Contraction pressure recorded using an intrauterine catheter Replot of part of a figure in reference [6].

Pre-delivery, the fetus and placenta are totally enveloped in the uterus (Figure 2a). If the uterine muscle contracts, all the tissue contained in the uterus will be subjected to the same additional raised pressure. For example, venous pressure in a fetus is typically $15 \mathrm{mmHg}$ [7]. Suppose a Braxton Hicks contraction [8] exerts a pressure of $20 \mathrm{mmHg}$. Then the fetal absolute venous pressure (measured from outside) will be $(15+20)$ or $35 \mathrm{mmHg}$. But the pressure in the tissue fluid surrounding all the local vessels would also have been raised by $20 \mathrm{mmHg}$, so the pressure distending a capillary would not have changed. Similarly, in the case shown in Figure 1, the absolute pressure measured via an intrauterine catheter rose to $100 \mathrm{mmHg}$, but so also would the pressure in the surrounding tissue fluid. The vascular distending pressure would have hardly changed. Some maternal blood may have been displaced from the maternal side of the placenta, but fetal vessels would not be damaged at this stage.

\section{Pressures during delivery}

Now the situation during parturition when the cervix has started to dilate will be considered during contractions (Figures $\mathbf{2 b}$ and $\mathbf{2 c}$ ). The scalp has lost the support of the uterus, it is exposed to air pressure only. Tissue fluid pressure under the newly exposed scalp surface will be virtually zero (ambient air pressure). However, the capillaries passing through this region are still connected to vessels in the fetus and so their lumens are at uterine contraction pressure. Capillary walls will be subjected to the full uterine contraction pressure. This may rupture some cerebral vessels, depriving local neurons of circulation, forming a diffuse injury. These ruptures will be obvious at autopsy and do not form part of HNE.

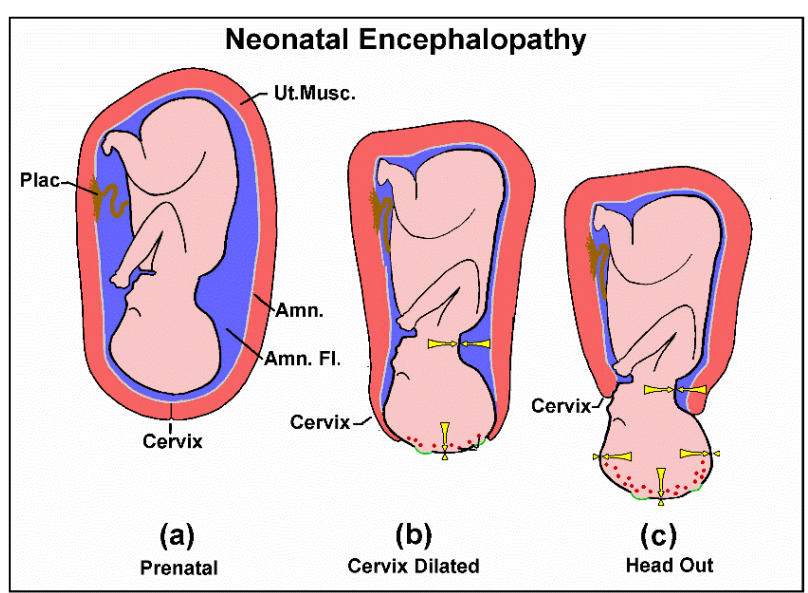

Figure 2 Contraction pressure distributions. Amn: Amnion; Amn. Fl.: Amniotic Fluid; Plac.: Placenta; Ut. Musc: Uterine Muscle; Yellow arrows represent vascular pressure at that position, Arrow heads represent atmospheric pressure at that position.

\section{Flooding}

It is normal for fluid to move through the walls of capillaries. The mean pressure in the lumen of typical capillaries is similar to that in the surrounding interstitium, slightly higher at the arteriolar end, slightly lower at the venule end. This facilitates a local fluid flow bringing oxygen to cells in the interstitium and carrying carbon dioxide away. Normally this pressure difference is only a few $\mathrm{mm} \mathrm{Hg}$, but, as previously indicated, during contractions pressure in cerebral capillaries may be 100 $\mathrm{mm} \mathrm{Hg}$ while they are surrounded by tissue fluid at $20 \mathrm{mmHg}$ pressure, a transmural pressure of $80 \mathrm{~mm} \mathrm{Hg}$. Such a high transmural capillary pressure, together with the increased permeability of the stretched walls, will rapidly force excessive volumes of plasma out into the surrounding interstitium.

\section{Phase II: Hypoxic-Ischemia and Treatment}

\section{Capillary constriction}

Constriction of capillaries will occur, not by the action of precapillary sphincters but by hydrostatic pressure in the tissue fluid. Figure 3 illustrates this process. Red signifies blood vessels and blue the interstitium surrounding them. Consider them to be capillaries fed from the left and drained to the right (Diagramatic, not to any scale).

Figure $3 a$ represents four adjacent capillaries in late pregnancy. Capillary $B$ is considered to have weaker and more porous walls compared to capillaries $A$ and $C$. This need not have any effect at normal fetal blood pressures and flows.

Figure $\mathbf{3 b}$ represents the situation during a contraction in early labor. The high lumen pressure is causing all of them to leak fluid (represented by white spots) especially capillary B. 
Capillary B leaks faster both because its nature is more permeable and because its walls are stretched. Between contractions, fluid will be driven back into the capillaries by the much smaller edemous pressure, so fluid will accumulate in the local interstitium, building up local edemous pressure.

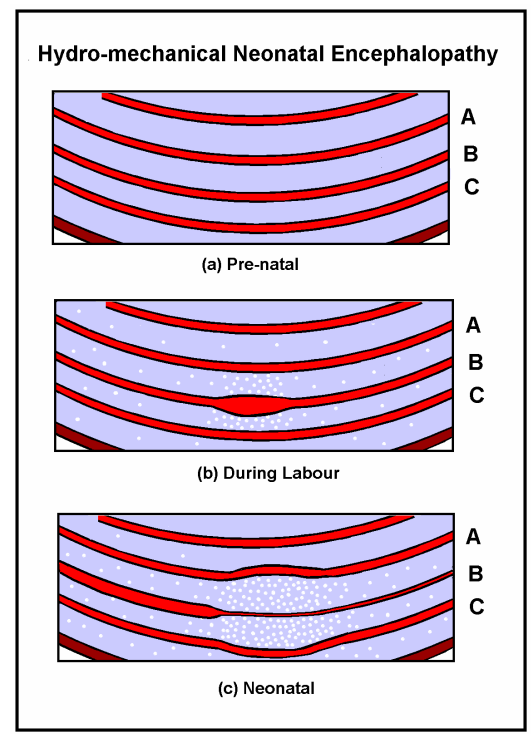

Figure 3 Stages in the development of temporary ischemia. (a) Representation of four adjacent cerebral capillaries during pregnancy, (b) Fluid escape through capillary walls during a contraction; with weak section in capillary $B,(c)$ Local high interstitial pressure compressing capillary B.

Figure $3 c$ represents the situation after delivery. The accumulated fluid around capillary $B$ has caused the local interstitium to swell and deflect capillaries A and C. Capillary B is surrounded by pressurised interstitium. This locally elevated interstitial pressure will constrict vessels by compressing them. Downstream of such constrictions (to the right in the figure) blood flow will be reduced, and upstream, flow will be redirected to unobstructed paths impairing delivery of oxygen and removal of carbon dioxide.

Thus, during contractions plasma forced through vessel walls into their surrounding interstitium will form highpressure cuffs around vessels. Between uterine contractions, and following delivery, this additional intrauterine pressure component will no longer be there. The cuffs will then press on the capillaries, narrowing them, and so restricting flow through them, causing local Ischemia.

\section{Ischemia}

Quite small compressions will make significant increases in flow resistance. The relationship between driving pressure and flow in a rigid tube can be described by a form of the Poiseuille Equation below [9]:

$$
Q=K \times D^{4} \times P
$$

Where $\mathrm{Q}$ is the flow rate, $\mathrm{K}$ is a constant describing the tube, and $P$ is the pressure driving the flow in a rigid tube, diameter $D$, can be described.

Capillaries are not rigid nor are they necessarily circular, but the fundamental approximation that for a given driving pressure flow through a tube varies as the fourth power of its radius, $10^{4}$, is valid. This means that quite small reductions in radius make a big difference in fluid flow resistance. If interstitional pressure reduces capillary radius by $10 \%$, flow resistance will increase by roughly $50 \%$. If the diameter is halved, flow will will be reduced to $6 \%$ of original. The magnitude of such flow reductions will vary from vessel to vessel producing a diffuse pattern of injury, whose local magnitude will depend both on the strength of the contractions and the maturity of the vasculature. Neighbouring vessels may be unaffected.

This Ischemia and consequential tissue hypoxia is only temporary, normoxia will return when the excess interstitial fluid disperses and its pressure on capillaries is removed [6]

\section{Therapeutic hypothermia}

The protective action of hypothermia will occur during the second phase of HNE. It involves keeping neurons alive, though not necessarily functional, in the temporary ischemic conditions arising following delivery. Hypothermia reduces metabolic rate by $7-9 \%$ per $1^{\circ} \mathrm{C}$ core temperature reduction [10], with parallel deceases in oxygen consumption and carbon dioxide production. Empirically, cooling works, but the optimum cooling temperature range and duration have yet to be determined. The following is based on the HydroMechanical concept of insult arising during delivery.

Temperature cooling range: Kerenyi et al. [10] studied systemic effects of the temperature when piglets were cooled during therapeutic hypothermia. They found that there was no difference in the relative risk of any neurodevelopmental outcome among piglets who received hypothermia with target core temperature $>34^{\circ} \mathrm{C}$ as compared to the control group. Therefore, the optimal temperature for neural rescue was likely to lie at some point below $34^{\circ} \mathrm{C}$. They also found more fatalities wth cooling to $30^{\circ} \mathrm{C}$ compared to normothermia or cooling to $35^{\circ} \mathrm{C}$. There was considerable risk of cardiac irregularities, more cardiac arrhythmias.

However, cooling to $33-34^{\circ} \mathrm{C}$ showed no physiological or clinical differences. This hypothetical hydro-mechanical model suggests that the requirement is to balance the benefits of slowing neurological metabolism against the hazards of slowing that of the heart sufficiently to affect its pacemaker.

Timing: After delivery the extravased fluid will start to drain away, and the vulnerable vessels will start to reopen. This is a complex phase because restoration of normal circulation is taking place while injury cascades have been triggered. This drainage time will vary from vessel to vessel because the vessels required to carry away the excess fluid are the same ones in which flow is restricted. 
It would seem important to maintain cooling until more normal pressures have returned to the vulnerable regions. The 72-hour period set in the TOBY study [5] seems to have been adequate on an empirical basis.

Summarizing: If this Hydro-mechanical Neonatal Encephalopathy hypothesis is valid-

1. Phase $1 \mathrm{HNE}$ in an individual infant can only occur during labour. It requires the excessive vascular pressures generated during uterine contractions. It cannot recur postnatally.

2. Local hypoxic stress in the cerebrum may begin in late stage one labour as the cervix starts to dilate.

3. Fluid is expelled through the walls of venules and capillaries by their excessive lumen pressure during contractions, raising local tissue fluid pressure.

4. Between contractions and in the neonatal period, this locally raised tissue fluid pressure may temporarily constrict venules, capillaries or even arterioles.

5. This temporary reduction of circulation will last until the excess fluid in the local interstitium surrounding vessels has dispersed, allowing the vessels to reopen.

6. Hypothermia works by enabling neurons to survive this temporary reduction of blood flow (ischemia) by reducing their metabolic activity.

7. Optimum cooling $\left(33-34^{\circ} \mathrm{C}\right)$ lies in the small range of temperature over which neural metabolic activity is reduced but cardiac pacemaker activity is not affected.

\section{Discussion}

The therapeutic benefit of hypothermia on this form of Hypoxic-Ischemic Encephalopathy lies in reducing neuronal activity and metabolic demands to keep neurons alive until local interstitial pressures have subsided, and normal blood flow is restored. It should be started as early as possible to conserve cell reserves.

\section{Permanence}

Analysis of neurological outcomes of various studies at 18 months of age [11] showed that treatment with hypothermia was consistently associated with an increased rate of normal survival. Among infants who survived to 18 months of age, those treated with hypothermia had significantly lower rates of severe disability, cerebral palsy, severe neuromotor delay, severe neurodevelopmental delay and blindness. This is consistent with the HME concept that conditions for the primary injury (vessel lumen at uterine contraction pressure, surrounded by tissue fluid at zero pressure) only exist relatively briefly. It starts late in the First Stage of labor and lasts while the head emerges but only until the body is free of uterine pressure.

Further damage may follow as a result of the hypoxia and ischemic injury, but the accumulation of expressed fluid itself cannot occur without access to the excessive intrauterine pressure.

\section{Indicated treatment}

Once recognised the infant should be cooled as soon as possible to conserve nutrients and slow down metabolism to match the meagre oxygen supply. Continue until the local tissue fluid accumulations have dispersed and normal capillary circulation has been restored.

\section{Conclusion}

1. There exists a form of Neonatal Encephalopathy that is entirely mechanical in origin. It results in temporary cerebral ischemia.

2. Hypothermia acts by reducing neural metabolic rate, to favour neural survival during this ischemic period.

3. Hydro-Mechanical Encephalopathy can only arise during delivery.

4. Hydro-Mechanical Encephalopathy is unrelated to previous events in that pregnancy.

\section{References}

1. Lally P, Pauliah S, Montaldo P, Chaban B, Oliveira V, et al. (2015) Magnetic Resonance Biomarkers in Neonatal Encephalopathy (MARBLE): a prospective multicountry study. BMJ Open 5: e008912.

2. Wikipedia (2017) Perinatal asphyxia. Wikipedia.

3. Battin M (2017) Neonatal Encephalopathy, Backgound. Newborn Services Clinical Guideline (Auckland Hospital) [http:// www.adhb.govt.nz/newborn/Guidelines/Neurology/NE.htm]

4. Zanelli SA, RosenKrantz T (2016) Hypoxic-Ischemic Encephalopathy. Medscape [https://emedicine.medscape.com/ article/973501-overview].

5. Azzopardi D, Strohm B, Edwards D, Dyet L, Haliday HL, et al. (2009) Moderate Hypothermia to Treat Perinatal Asphyxial Encepphalopathy. N Eng J Med 361: 1349-1358.

6. O'Connell NG, Smith CV (2015) Intrauterine Pressure Catheter Placement. Medscape [emedicine.medscape.com/article/ 1998044-overview]

7. Ganong WF (1987) Dynamics of blood \& lymph flow. In: Ganong WF, editor. Review of medical physiology (13edn) Connecticut: Prentice-Hall International Inc, pp: 477-492.

8. Wikipedia (2017) Braxton Hicks Contractions. Wikipedia.

9. OpenAnesthesia Poiseuille's Law: IV fluids International Anesthesia Research Society [https://www.openanesthesia.org/ poiseuilles_law_iv_fluids].

10. Kerenyi A, Kelen D, Faulkner SD, Bainbridge A, Chandrasekare $M$, et al. (2012) Systemic effects of whole-body cooling to $35^{\circ} \mathrm{C}$, $33.5^{\circ} \mathrm{C}$ and $35^{\circ} \mathrm{C}$ in a piglet model of perintal asphyxia:implications for therapeutic hypothermia. Pediatric Research 71: 573-582.

11. Edwards D, Brocklhurst P, Gunn AJ, Halliday H, Juszcak E, et al. (2010) Neurological outcomes at 18 months of age after 
moderate hypothermia for perinatal hypoxic ischaemic encephalopathy: synthesis and meta-analysis of trial data. BMJ 340: 363. 